\title{
ROLE OF IMAGING IN ANTENATAL DIAGNOSIS OF BLADDER EXSTROPHY - EPISPADIAS COMPLEX
}

Rahul Jain ${ }^{1}$, Bhawna Solanki², Savita Shekhawat ${ }^{3}$, Megha Saini ${ }^{4}$, Hemant Mishra ${ }^{5}$.

1. III Year PG Resident, Department of Radiodiagnosis, Imaging and Paediatrics, Mahatma Gandhi Medical College \& Hospital, MGUMST.

2. III Year PG Resident, Department of Radiodiagnosis, Imaging and Paediatrics, Mahatma Gandhi Medical College \& Hospital, MGUMST.

3. III Year PG Resident, Department of Radiodiagnosis, Imaging and Paediatrics, Mahatma Gandhi Medical College \& Hospital, MGUMST.

4. Senior Resident, Department of Radiodiagnosis, Imaging and Paediatrics, Mahatma Gandhi Medical College \& Hospital, MGUMST.

5. Professor \& HOD, Department of Radiodiagnosis, Imaging and Paediatrics, Mahatma Gandhi Medical College \& Hospital, MGUMST.

\section{CORRESPONDING AUTHOR}

Dr. Bhawna Solanki,

Department of Radiology,

Mahatma Gandhi Hospital,

Sitapura, Jaipur (302022), Rajasthan.

Email- drbhawna2901@yahoo.co.in

\section{HOW TO CITE THIS ARTICLE:}

Rahul Jain, Bhawna Solanki, Savita Shekhawat, Megha Saini, Hemant Mishra. "Role of Imaging in Antenatal Diagnosis of Bladder Exstrophy - Epispadias Complex". Journal of Evolution of Medical and Dental Sciences 2013; Vol2, Issue 23, June 10; Page: 4223-4228.

ABSTRACT: Bladder exstrophy-epispadias complex is a rare congenital anomaly. A higher occurrence in males compared to females is observed. It occurs due to failure of the abdominal wall closure during fetal development and results in protrusion of the posterior bladder wall through the lower abdominal wall. It involves a spectrum of anomalies of the lower abdominal wall, bladder, anterior bony pelvis and external genitalia. Here, we report a case of antenatal diagnosis of bladder exostrophy -epispadias complex in a seven months pregnant patient who came to us for routine antenatal sonography. On fetal MRI, our findings were confirmed. Patient was followed and normal vaginal delivery was done.

KEY WORDS: exstrophy bladder, exstrophy cloaca, Epispadias.

INTRODUCTION: The Exstrophy-epispadias complex (EEC) is the most serious form of abdominal midline malformation. The characteristic defects of the EEC involve the urinary system, the musculoskeletal system, the pelvis, the pelvic floor, the abdominal wall, the genitalia and sometimes the spine and the anus. ${ }^{1}$

The EEC covers a spectrum with different severity levels, ranging from epispadias (E) representing the mildest form, with lower and upper fissure, to the full picture of classical bladder exstrophy (CEB), and exstrophy of the cloaca (EC) - often also referred to as OEIS (omphalocele, 
exstrophy, imperforate anus and spinal defects) complex - as the most severe form. Varying data have been reported on the incidence of EEC with respect to different subtypes, ethnicity, and sex ratio. The incidence of classic bladder exstrophy varies from 2.1 to 4 per 100,000 live births, according to different reports. It appears to be increased with lower maternal age, higher parity, and Caucasian race. Summarized data from multiple surveys suggest a 2.4:1 male-to-female ratio, although population-based studies have reported a similar incidence between males and females. The reported incidence of epispadias varies according to the geographic distribution, but 2.4 per 100,000 , as reported by the International Clearinghouse for Birth Defects Monitoring System, is commonly accepted ${ }^{2}$.

Diagnosis at birth is made on the basis of the clinical presentation but EEC may be detected prenatally by ultrasound from repeated non-visualization of a normally filled fetal bladder. Counseling should be provided to parents but, due to a favourable outcome, termination of the pregnancy is no longer recommended. Management is primarily surgical.

CASE REPORT: A 22 years old, Gravida 2, para 1 came to us for routine antenatal fetal well being sonography. Patient had seven months amenorrhea. On sonography, single live fetus with cephalic presentation with average maturity of 30 weeks was seen. There was evidence of severe oligohydramnios (AFI-3cm). There was a cystic mass of 15.2 X $6.8 \mathrm{~cm}$ with fluid-fluid level outside the fetal pelvis communicating with the urinary bladder. There was evidence of mild hydronephrosis on left side with PUJ narrowing /obstruction. On sonography, diagnosis of bladder exostrophy, left PUJ obstruction \& oligohydramnios was made. Fetal MR revealed a defect in the anterior abdominal wall of fetus with protrusion of bladder through this defect with mild to moderate Left hydronephrosis. Normal vaginal delivery was done. Baby was a male Child with Undescended Testis, a triangle - shaped defect in abdomen \& visible mucosa of bladder which was bright pink with outwardly rotated legs \& feet, and cranially displaced umbilicus. The baby had Short penis with urethral opening along dorsal surface (Epispadias). Our findings on antenatal ultrasonography and Fetal MR were confirmed.

DISCUSSION: A bladder exostrophy refers to a herniation of the urinary bladder through an anterior abdominal wall defect. Embryologically, many theories of this anomaly have been published but most accepted is of Marshall and Muecke which describes that there is basic defect of overdevelopment of cloacal membrane which prevent medial migration of mesenchymal tissue, therefore proper development of anterior abdominal wall doesn't occur ${ }^{3}$. Ambrose SS and O'Brien postulated that the genital hillocks with the fusion in the midline below rather than above cloacal membrane results in exostrophy of bladder with episadias ${ }^{4}$. Boyadjiev et al postulated the CASPR 3 gene on chromosome 9 is associated with this complex 5 .

Exostrophy Bladder can occur to variable severity ranging from epispadias (E) to classical bladder exstrophy (CEB) and exstrophy of the cloaca (EC). Depending on severity, it may involve the urinary system, musculoskeletal system, pelvis, pelvic floor, abdominal wall, genitalia, and sometimes the spine and anus. EEC can be subdivided into "classic" or "typical" forms of EEC (E, CEB, and EC) and "atypical" forms of the EEC (duplicated exstrophy, covered exstrophy and pseudoexstrophy). It has a higher occurrence in males compared to females with 2.4:1 male-to-female ratio. Associated anomalies are Urological anomalies such as ureteropelvic junction obstruction, 
ectopic pelvic kidney, horseshoe kidney, renal hyo- or agenesis, megaureter, ureteral ectopy and ureterocele) are present in about one third of all EEC cases, predominantly in the EC population 1. However, a $100 \%$ prevalence of bilateral vesicoureteral reflux due to a developmental failure of the ureterovesical junction is also seen. Spinal and orthopedic anomalies occur in about $7 \%$ of cases, whereas a heterogeneous group of congenital spinal anomalies resulting from defective closure of the neural tube early in fetal life and anomalous development of the caudal cell mass can be confirmed with magnetic resonance imaging (MRI) in nearly $100 \%$ of EC patients. Therefore, newborn EC patients should have spinal ultrasound and radiographs to define the individual spinal abnormalities ranging from hemivertebra to myelomeningocele. In addition, a neurological component must be kept in mind in EC in respect to bladder function, lower extremity function and erectile capacity ${ }^{6-8}$. Mainly in EC, skeletal and limb anomalies (clubfoot deformities, absence of feet, tibial or fibular deformities, and hip dislocations) are commonly seen ${ }^{1}$. Associated Gastrointestinal tract anomalies are predominantly associated with EC, and will seldom be present in CEB or E. In addition to a common hindgut remnant of varying size, omphaloceles are found in $88-100 \%$ of cases in EC. Gastrointestinal malrotation or duplication, as well as short bowel syndrome, can be seen in up to $46 \%$ of cases $^{1}$. Gynecological anomalies are external female genitalia, the cervix inserts in most cases low down at the superior vaginal wall close to the introitus ${ }^{1-9}$. Müllerian anomalies are quite common in EC; respectively, duplication of the vagina and uterus, as well as vaginal agenesis have been reported ${ }^{1}$

Due to high-resolution real-time ultrasound, prenatal diagnosis of EEC is usually possible between the 15th and 32nd week of gestation. The index finding is the non-visualization of a normally filled fetal bladder during repeated careful ultrasound examinations. A low-set umbilicus, a wide ramus pubis, diminutive genitalia and a lower abdominal mass were summarized as diagnostic key factors for EEC diagnosis, in addition to the absent bladder filling 1 . Another typical feature is a wavy cord-like segment of soft tissue protruding from the anterior abdominal wall, just below the umbilical cord insertion, strongly resembling the trunk of an elephant 1,10,11.

MR has an important role in prenatal diagnosis of bladder exostrophy. The MRI defined findings in EEC as a lower abdominal mass prolapsing below the umbilical vessels, having the ureters ending on it in an anterior position. A cloacal malformation or a cloacal exstrophy could be excluded, as well as other accompanying spinal abnormalities. The renal system and oligohydramnios could be well documented ${ }^{12}$.

CONCLUSION: The management of bladder exstrophy has improved to provide better quality of life of such children. Role of imaging in antenatal diagnosis of BEEC is of paramount importance to prepare the parents and surgeon for appropriate surgical management. High resolution Ultrasound and MRI can adequately delineate the extent of abnormality and co-existent anomalies. After sonographic and MR diagnosis of EEC has been established, adequate counselling of parents must be done.

\section{REFERENCES:}

1. Gearhart JP: The bladder exstrophy-epispadias-cloacal exstrophy complex. In Pediatric Urology Volume Chapter 32. Edited by: Gearhart JP, Rink RC, Mouriquand PDE. Philadelphia: W. B. Saunders Co; 2001:511-546. 


\section{CASE REPORT}

2. Nima Baradaran and John P. Gearhart: Bladder Exstrophy-Epispadias- Cloacal Exstrophy Complex: A Contemporary Overview Neo Reviews 2010;11;e705-e713 DOI : 10.1542/neo.1112 -e705

3. Muecke EC: The role of the cloacal membrane in exstrophy: The first successful experimental study. Journal of Urology, 1964; 92:659-667.

4. Ambrose SS, O'Brien DP: Surgical embryology of the exstrophy-epispadias complex. Surgical Clinics of North America, 1974; 54: 1379-1390.

5. Boyadjiev SA, Dodson JL, Radford CL, Ashrafi GH, Beaty TH, Mathews RI, Broman KW, Gearhart JP: Clinical and molecular characterization of the bladder exstrophy-epispadias complex: analysis of 232 families. BJU Int 2004, 94:1337-1343.

6. Rösch WH, Hanisch E, Hagemann M, Neuhuber WL: The Characteristic innervation pattern of the urinary bladder in particular forms of exstrophy-epispadias-complex. BJU 2001, 87(Suppl 1):30.

7. Rösch W, Christl A, Strauß B, Schrott KM, Neuhuber WL: Comparison of preoperative innervation pattern and postre constructive urodynamics in extrophy-epispadias-complex. Urol Int 1997, 59:6-15.

8. Schober JM, Carmichael PA, Hines M, Ransley PG: The ultimate challenge of cloacal exstrophy. $J$ Urol 2002, 167(1):300-304.

9. Woodhouse CRJ, Hinsch R: The anatomy and reconstruction ofthe adult female genitalia in classical exstrophy. BJU 1997, 79:618-622

10. Della Monica M, Nazzaro A, Lonardo F, Ferrara G, Di Blasi A, Scarano G: Prenatal ultrasound diagnosis of cloacal exstrophy associated with myelocystocele complex by the 'elephant trunk like' image and review of the literature. Prenat Diagn 2005, 25:394-397

11. Cromie WJ, Lee K, Houde K, Holmes L: Implications of prenatal ultrasound screening in the incidence of major genitourinary malformations. J Urol 2001, 165(5):1677-80.

12. Goldman S, Szejnfeld PO, Rondon A, Francisco VV, Bacelar H, Leslie B, Barroso U Jr, Ortiz V, Macedo A Jr J Pediatr Urol. 2013 Feb;9(1):3-6. doi: 10.1016/j.jpurol.2012.06.018. Epub 2012 Jul 26. 


\section{CASE REPORT}

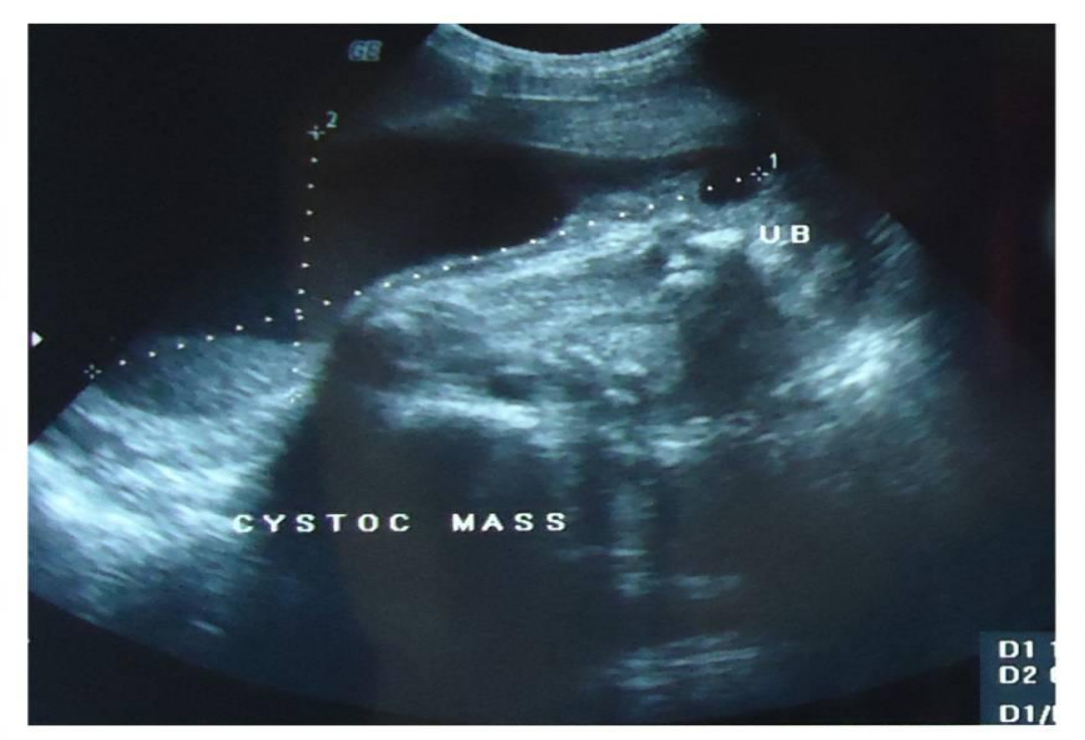

FIGURE 1: A Cystic Mass of 15.2 x $6.8 \mathrm{~cm}$ with Fluid Level is seen outside the pelvis which is communicating with the Urinary Bladder

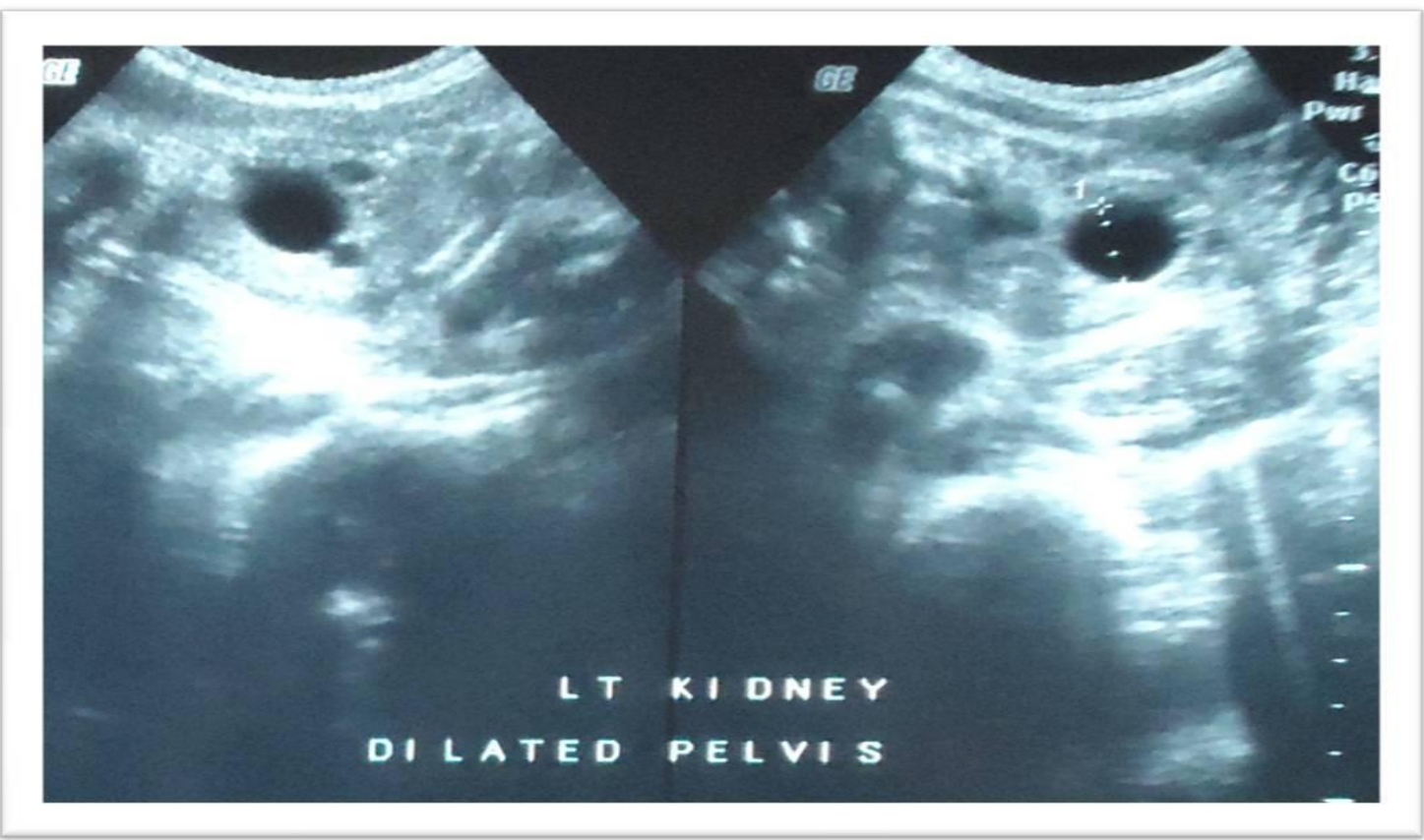

FIGURE 2: Pelvis of Left Kidney is dilated \& show abrupt cut off (? PUJ Narrowing) 


\section{CASE REPORT}

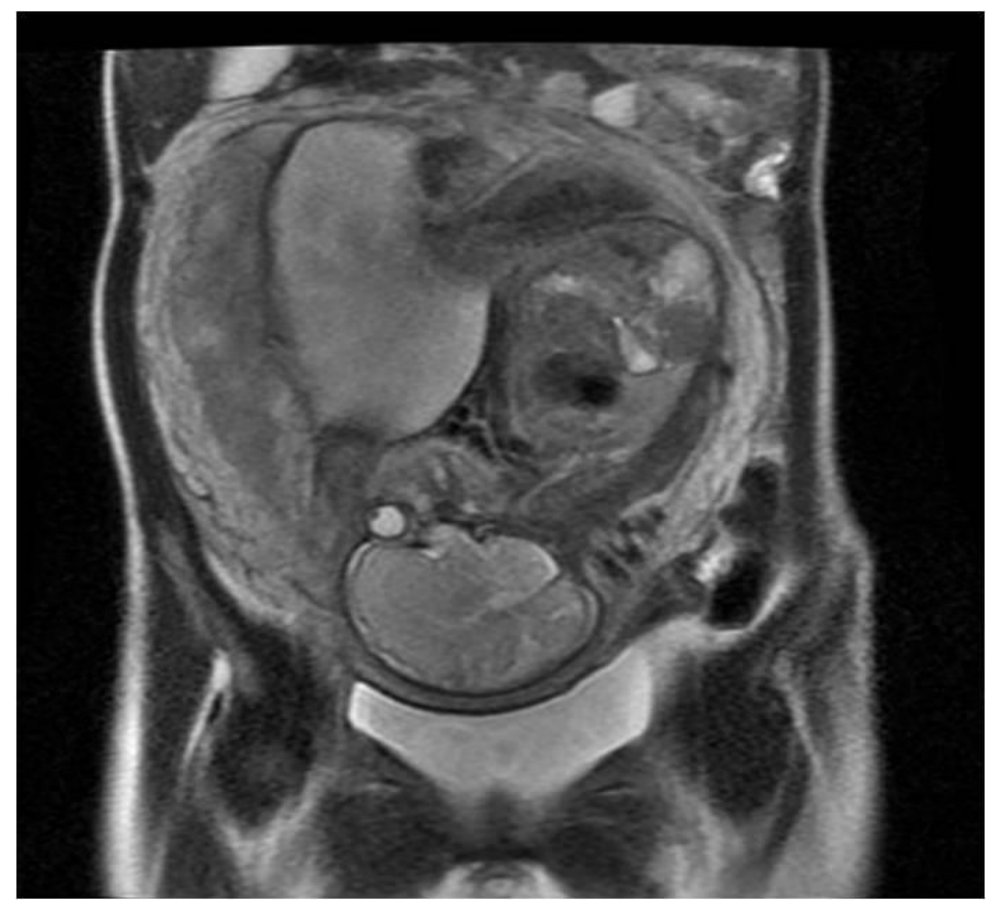

FIGURE 3: T2W Oblique sagittal view of fetus shows large well defined cystic lesion seen lying extra abdominally communicating with the urinary bladder.

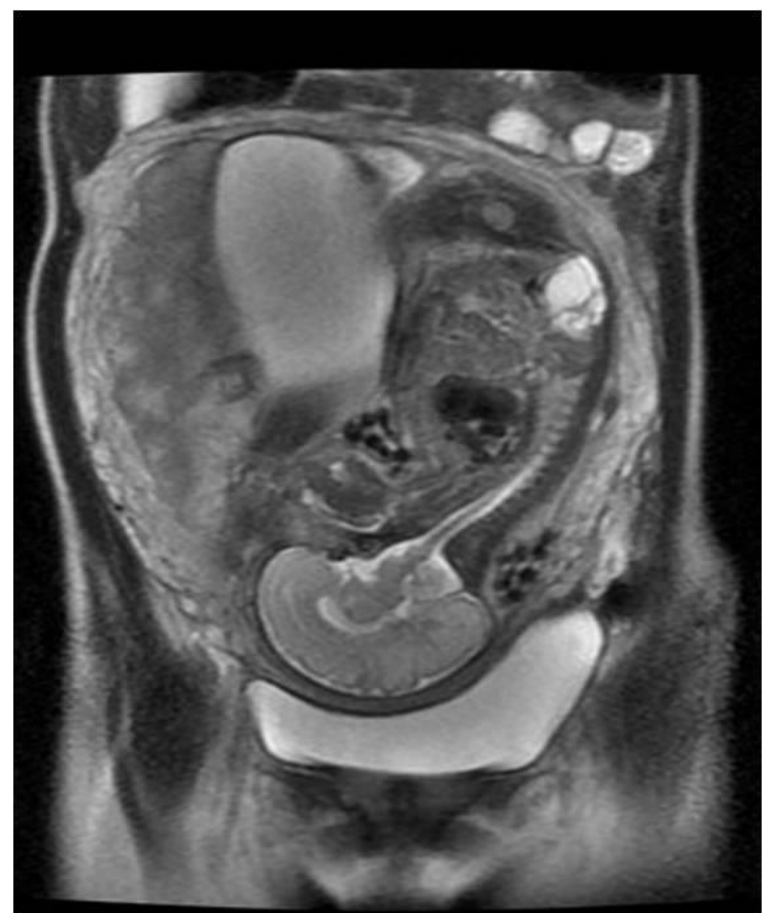

FIG- 4

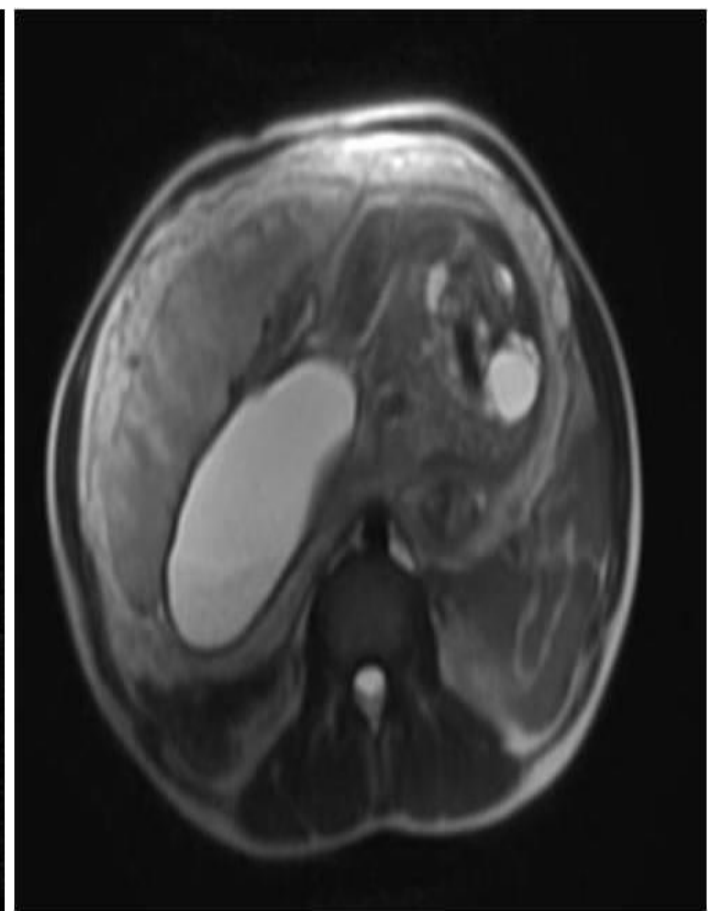

FIG 5

FIGURES 4, 5: T2W Sagittal and axial images shows hydronephrotic left kidney with dilated renal pelvis in addition to large extra abdominal cystic swelling. 